\title{
Differential Expression of Long Noncoding RNA in Primary and Recurrent Nasopharyngeal Carcinoma
}

\author{
Wei Gao, Jimmy Yu-Wai Chan, and Thian-Sze Wong \\ Department of Surgery, LKS Faculty of Medicine, The University of Hong Kong, Queen Mary Hospital, \\ 102 Pokfulam Road, Hong Kong, Hong Kong
}

Correspondence should be addressed to Thian-Sze Wong; thiansze@graduate.hku.hk

Received 11 February 2014; Accepted 20 March 2014; Published 14 April 2014

Academic Editor: Thean Hock Tang

Copyright (C) 2014 Wei Gao et al. This is an open access article distributed under the Creative Commons Attribution License, which permits unrestricted use, distribution, and reproduction in any medium, provided the original work is properly cited.

Background. Recent studies suggested that non-protein-coding genes are implicated in the tumorigenic process of nasopharyngeal carcinoma (NPC). In the present study, we aimed to identify the differentially expressed long noncoding RNA (lncRNA) using data available in the public domain. Methods. Microarray data set GSE12452 was reannotated with ncFANs. Real-time quantitative PCR was used to quantify and validate the identified lncRNAs in NPC. Results. In primary NPC, upregulation of lnc-C22orf32-1, lncAL355149.1-1, and lnc-ZNF674-1 was observed. High levels of lnc-C22orf32-1 and lnc-AL355149.1-1 were significantly associated with the male patients. In addition, increased expression of lnc-C22orf32-1 and lnc-ZNF674-1 was associated with advanced tumor stages. Recurrent NPC displayed a distinctive lncRNA expression pattern. lnc-BCL2L11-3 was significantly increased in the recurrent NPC tissues. In addition, significant reduction of lnc-AL355149.1-1 and lnc-ZNF674-1 was observed in the recurrent NPC tissues. Conclusions. Our results demonstrated that it is feasible to identify the differentially expressed lncRNA in the microarray dataset by functional reannotation. The association of lncRNA with gender and tumor size implicated that lncRNA possibly plays a part in the pathogenesis of primary NPC. Further, the distinctive lncRNA identified in the recurrent NPC may reveal a distinctive development mechanism underlying tumor recurrence.

\section{Introduction}

Undifferentiated nasopharyngeal carcinoma (NPC) is prevalent in specific geographic localities including Southern China and East Asia, Southeast Asia, Greenland (native), Canada (Northwest Territories), and Alaska. Undifferentiated NPC is characterized by its close association with the virology of Epstein-Barr virus, a gamma-herpes DNA virus that infects more than $90 \%$ of world's population and is found in nearly all undifferentiated NPC cells [1]. According to the World Health Organization classification (WHO 2005), NPC could be classified based on the cancer differentiation status into type 1 keratinising squamous cell carcinoma and type 2 nonkeratinising carcinoma (differentiated and undifferentiated NPC). In endemic regions, the major histological type is type 2 NPC [2]. Early diagnosis of NPC is difficult as the cancer is developed from the epithelial lining around the ostium of the Eustachian tube in the lateral wall of the nasopharynx with close proximity to the skull [3]. Later diagnosis will usually lead to unsatisfactory treatment results and high incidence of local regional recurrence [4].

lncRNAs are non-protein-coding transcripts transcribed by either RNA Pol I, RNA Pol II, or RNA Pol III. Different from the protein-coding genes, lncRNA has distinct biological function in maintaining the cellular homeostasis [5]. Prensner and Chinnaiyan summarized our current knowledge on lncRNA in the pathogenesis and progression of human malignancies [6]. At present, we know that long or large intergenic ncRNAs, transcribed ultraconserved regions, pseudogenes, enhancer RNAs, antisense RNAs, and long stress-induced noncoding transcribes are involved in the development and tumorigenesis of cancers. The existence of these noncoding RNAs used to be regarded as transcriptional byproducts with no significant functions. Over the past decade, however, increasing evidence suggested that lncRNA could function as epigenetic regulators, modulators of gene expression, and protein degradation machinery as well as regulators of organelle biogenesis and subcellular trafficking [7]. 
Hence, further understanding of the aberrant expression pattern of lncRNA in cancers is essential.

Study on lnRNA deregulation in undifferentiated NPC remains raw. Recently, with the use of candidate gene approach, it was identified that HOX antisense intergenic RNA (HOTAIR), one of the first described lncRNAs transcribed in the HOXC locus at 12q13.13, was suggested to play a part in NPC. HOTAIR was implicated in cancer progression and metastasis. In primary NPC, high HOTAIR expression was specific to the primary NPC and was absent in the normal nasopharyngeal epithelia. HOTAIR expression level was linked with the tumor size, nodal status, and distant metastasis. Further, patients with high HOTAIR levels will have poor clinical outcome with tumor recurrence and distant metastasis $[8,9]$.

High-throughput microarray was originally designed to detect and quantify the protein-coding genes. However, it is now noted that many of the probes on the microarray also match with the sequence of lncRNA. By rematching and reannotation of the existing microarray datasets, the results obtained from the microarray experiments could be used for lncRNA analysis concurrently. For example, with the use of Affymetrix U133A and B microarrays, it has been demonstrated that the microarray data can be reannotated for lncRNA expression analysis [10]. Here, we explored the differential lncRNA expression patterns in nasopharyngeal carcinoma (NPC) based on the high-throughput NPC dataset in the public domain using the functional reannotation service provided by noncoding RNA Function ANnotation server (ncFANs) [11]. The identified lncRNAs were validated in both primary and recurrent NPC samples.

\section{Materials and Methods}

2.1. Dataset. Microarray data set GSE12452 containing 41 microarray data (http://www.ncbi.nlm.nih.gov/geo/query/ acc.cgi?acc=GSE12452) was collected from Gene Expression Omnibus (GEO). The dataset contains $31 \mathrm{NPC}$ and 10 normal nasopharyngeal tissue samples examined with Human Genome U133 Plus 2.0 Array (HG-U133 Plus 2) from Affymetrix [12]. The tissues were confirmed histologically before the microarray experiment. Tumor tissue was defined by the positive stain for both cytokeratins and EBV EBERs using immunohistochemical staining. All the samples were processed by laser-captured microdissection to enrich the epithelial cells before total RNA extraction.

2.2. Patient Samples. Undifferentiated NPC tissue samples were obtained from Department of Surgery, The University of Hong Kong, Queen Mary Hospital, Hong Kong. Written consent of tissue donation for research purposes was obtained from patients before tissue collection. The protocol was approved by the IRB of the hospital (reference number UW 10-142). Tissue samples were stored at liquid nitrogen for transportation and stored at $-70^{\circ} \mathrm{C}$ before use. In total, nasopharyngeal tissue samples were collected from 42 patients with primary NPC, 33 patients with recurrent NPC, and 29 noncancer volunteers. The 42 patients with primary
NPC included 32 male and 10 female, with age ranging from 12 to 75 years. The 33 recurrent NPC patients included 24 male and 9 female with age ranging from 29 to 75 years. The 29 healthy volunteers included 20 male and 9 female, with ages ranging from 6 to 81 years.

\subsection{Reannotation and Selection of Differential Expressed} $\operatorname{lncRNA}$. The microarray data was preprocessed with the preprocessing program and reannotated with the Affymetrix CDF file from the web server of ncFANs (noncoding RNA Function ANnotation server). The data was normalized by MAS5.0 before lncRNAs annotation. Transcripts with length $<200$ were excluded [11]. Differentially expressed lncRNAs were selected using Student's $t$-test using $P$ value below 0.05 as cutoff.

2.4. RNA Extraction and Real-Time Quantitative RT-PCR Validation of the Microarray Results. Total RNA was extracted with TRIZOL reagent (Invitrogen) following the manufacturer's protocol. The cDNA product was generated using cDNA conversion kit (Invitrogen). Primers were designed with Primer3 provided by the Universal ProbeLibrary Assay Design Center (http://www.roche-applied-science.com/). The LNA-labelled probe was obtained from the Universal ProbeLibrary (UPL). The lncRNA transcript levels were quantified on LightCycler 480 (Roche Applied Science) and normalized with the GAPDH levels by $2^{-\Delta \mathrm{Ct}}$ method. All reactions were done in triplicate. Primer and probe sequences were shown in Table 1.

2.5. Statistical Analysis. Statistical analysis was performed with SPSS V16.0 (SPSS, Chicago, IL). The difference between primary NPC and controls was calculated using the MannWhitney $U$ test. The statistical difference between recurrent NPC and paired normal tissues was examined using Wilcoxon Signed Rank test. All the tests were two-sided. $P$ value $<0.05$ was considered statistically significant.

\section{Results}

3.1. Functional Annotation of IncRNA with ncFANs. The differentially expressed lncRNAs identified were listed in Table 2. Five lncRNAs were found to be differentially expressed in the nasopharyngeal carcinoma tissues in comparison with the normal nasopharyngeal epithelial tissues in the microarray data. The lncRNAs identified were lncC22orf32-1, lnc-TLR4-1, lnc-BCL2L11-3, Inc-AL355149.1-1, and lnc-ZNF674-1.

3.2. IncRNA Validation on Primary NPC Tissues and Normal Nasopharyngeal Epithelia. To validate the identified lncRNA, we performed real-time quantitative analysis on the candidate lncRNA in our cohort. First, we examined the fold changes in the primary nasopharyngeal carcinoma and normal nasopharyngeal tissues (Figure 1). Among the 5 candidate lncRNAs, lnc-C22orf32-1, lncTLR4-1, lnc-AL355149.11 , and lnc-ZNF674-1 demonstrated significant expression 
TABLe 1: Primer and probe sequences used in quantitative PCR.

\begin{tabular}{lcccc}
\hline Vega gene ID & LNCipedia gene ID & Forward primer $\left(5^{\prime}-3^{\prime}\right)$ & Reverse primer $\left(5^{\prime}-3^{\prime}\right)$ & UPL number $^{*}$ \\
\hline OTTHUMG00000153695 & lnc-BCL2L11-3 & agcagatgctgtgcctgata & cctttctcgacccagaagc & $\# 67$ \\
OTTHUMG00000002490 & lnc-AL355149.1-1 & gaaaactaggcgtctgggaac & caaacaatgggagcaagtcc & \#25 \\
OTTHUMG00000150697 & lnc-C22orf32-1 & tgctcatcttctgccacagt & agggcagtgatgaggaacc & \#38 \\
OTTHUMG00000021416 & lnc-ZNF674-1 & agcacttggccctaaagaga & aacatactggcccaaacagc & $\# 88$ \\
OTTHUMG00000020561 & lnc-TLR4-1 & ccacacaaatgggcaagaat & gcaaaatcctgaaggttcaaa & $\# 6$ \\
\hline
\end{tabular}

${ }^{*}$ Probe number in the universal probe library (UPL).

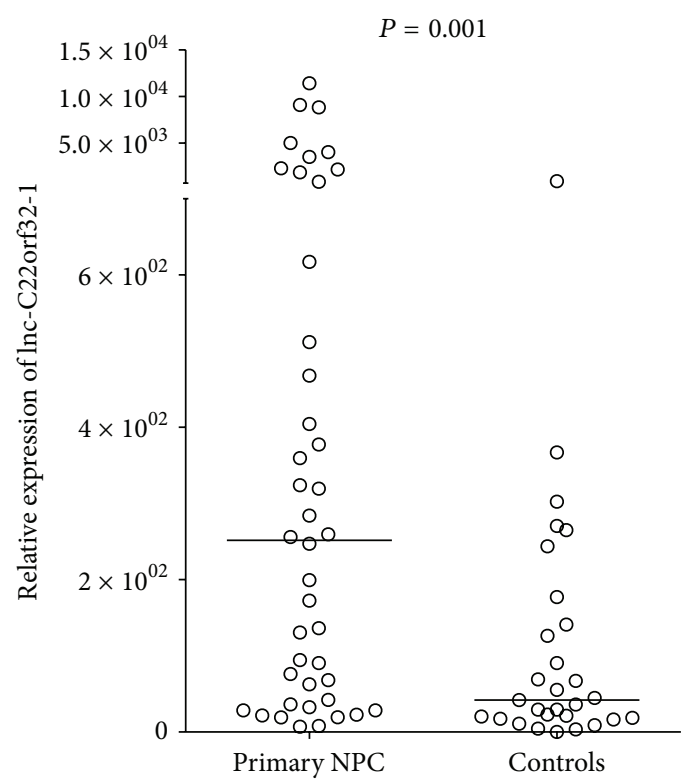

(a)

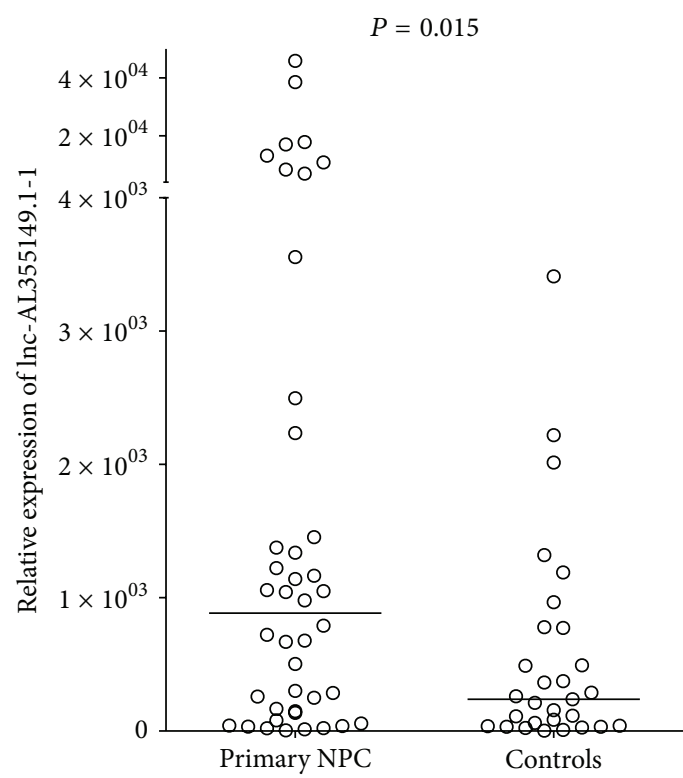

(c)

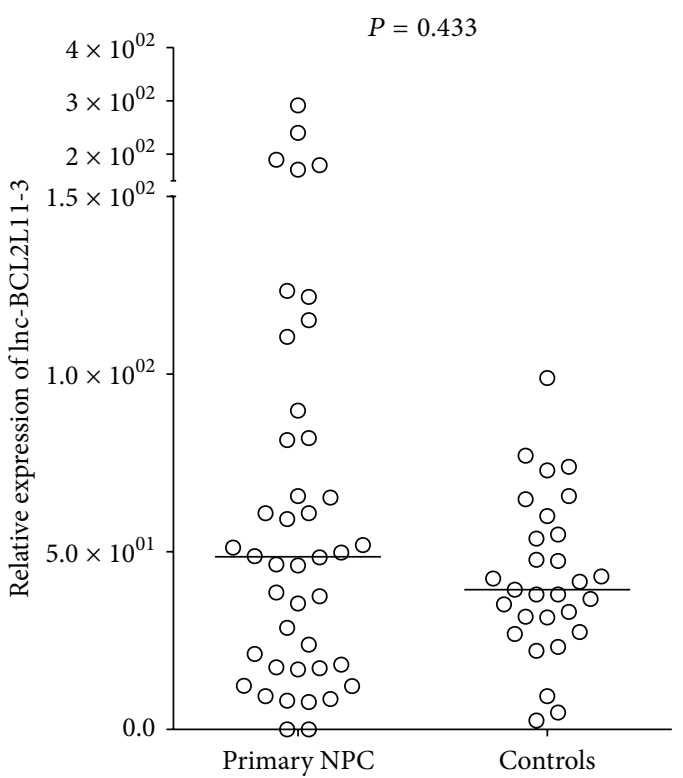

(b)

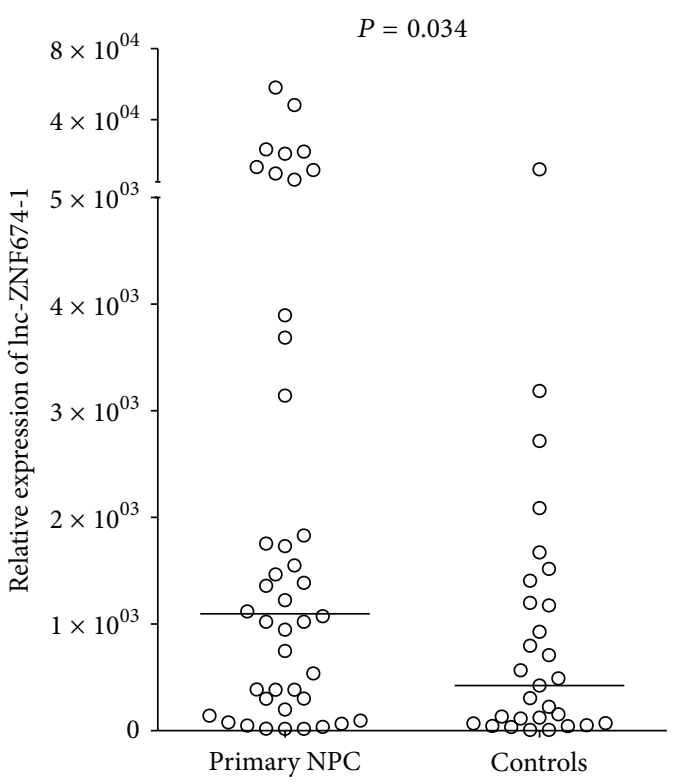

(d)

FIGURE 1: Relative expression levels of lncRNAs in tissue samples from patients with primary NPC and healthy controls. The relative expression levels were normalized to GAPDH by qPCR analysis. The difference between primary NPC and controls was calculated using the MannWhitney $U$ test. 


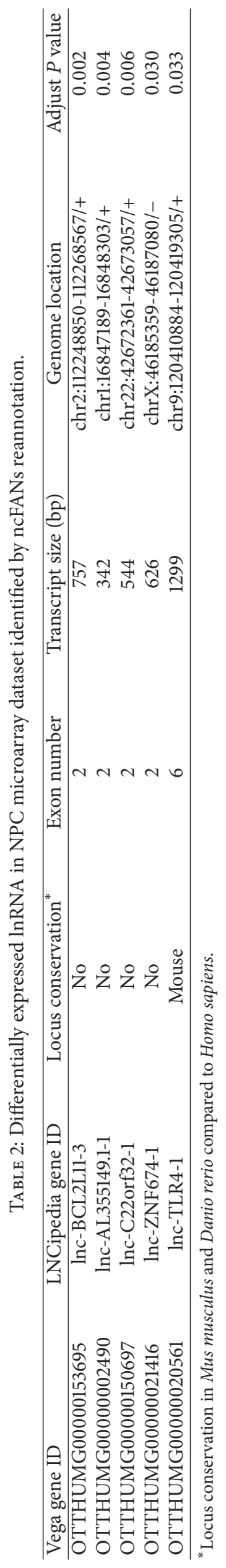




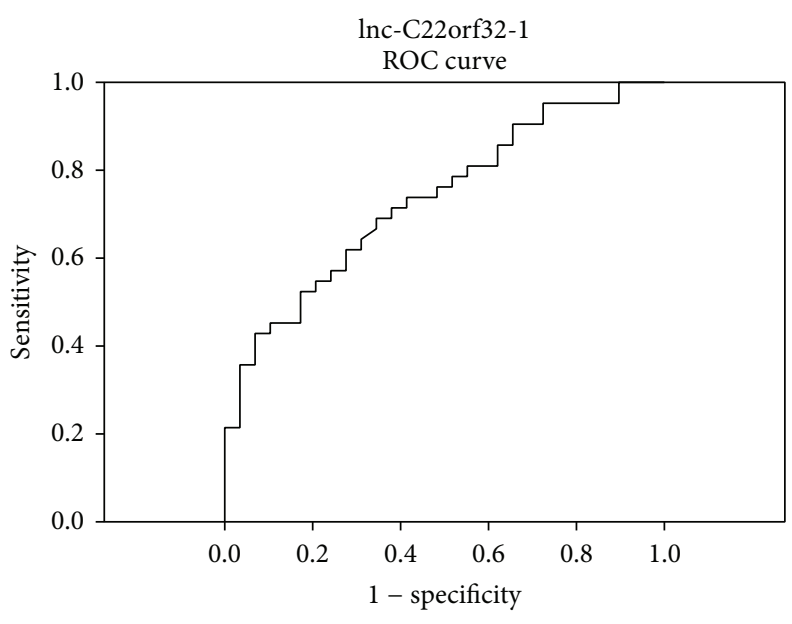

Area under curve $=0.734$

(a)

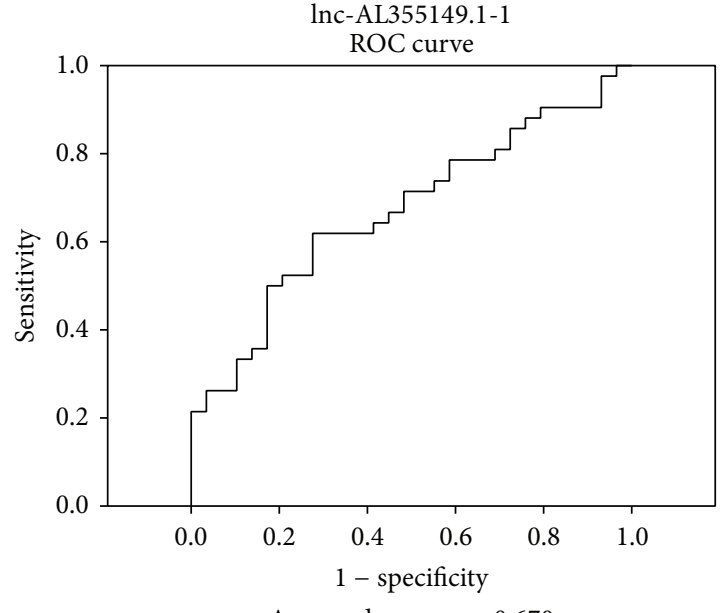

Area under curve $=0.670$

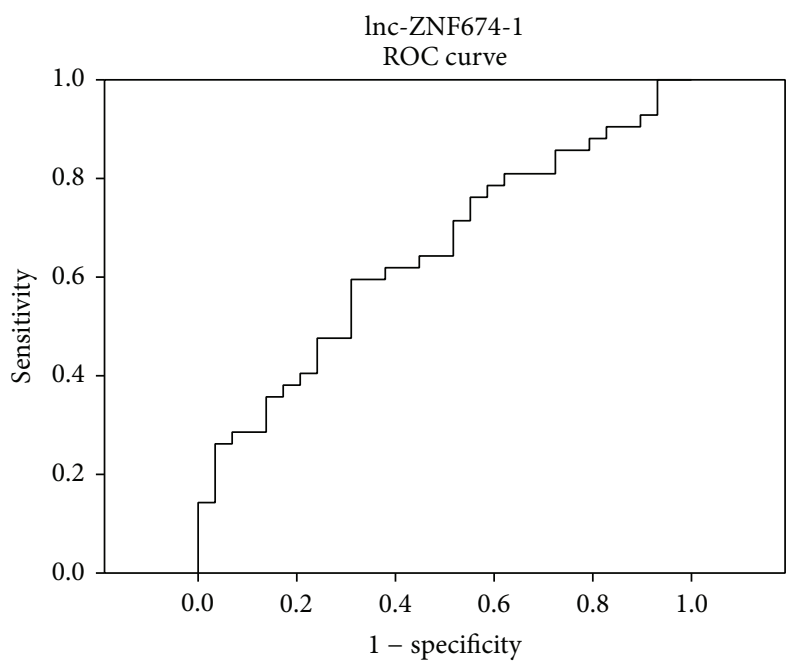

Area under curve $=0.649$

(c)

FIGURE 2: Receiver operating characteristic (ROC) curve analysis of lncRNAs in tissue samples from patients with primary NPC and healthy controls.

difference between the primary NPC and normal nasopharyngeal tissues.

Of the 4 lncRNAs, the expression difference of lncC22orf32-1 was highly significant $(P=0.001$, Mann-Whitney $U$ test). High lnc-C22orf32-1 was found in the primary NPC tissues in comparison with the normal controls (Figure 1). In ROC analysis, the AUC value of lnc-C22orf32-1 was 0.734 in differentiating NPC from the normal nasopharyngeal tissues (Figure 2); lnc-AL355149.1-1 and lnc-ZNF674-1 were also found to be overexpressed in the primary NPC tissues ( $P=$ 0.015 and 0.034 , respectively, Mann-Whitney $U$ test). The 3 lncRNAs above are detectable in all the tissues examined. In contrast, lnc-TLR4-1 was detectable in 21\% (9/42) patients with primary NPC and 66\% (19/29) healthy controls. In recurrent NPC, lnc-TLR4-1 was detectable in 27\% (9/33) tumor tissues and 15\% (5/33) paired normal tissues. Low copy number of lnc-TLR4-1 in tissue samples may account for the low detection rate. The expression pattern of lnc-TLR41 was not correlated with age or gender (data not shown). Since lnc-TLR4-1 was only expressed in a subset of NPC and normal nasopharyngeal tissues, it was not suitable for study of differential expression.

Next, we compared the expression level of the 3 universal expressed lncRNAs with the clinicopathological parameters of the primary NPC patients (Table 3). High levels of lncC22orf32-1 and lnc-AL355149.1-1 were significantly associated with the male patients (Figure 3(b)). In addition, increased expression of lnc-C22orf32-1 and lnc-ZNF674-1 was associated with advanced tumor stages (T2-4) in comparison with the early stage (T1) NPC (Figure 3(a)).

3.3. Differential IncRNAs Expression in Recurrent NPC and the Paired Normal Epithelia. We compared the lncRNA expression patterns with the paired normal tissues obtained from 
TABLE 3: Association of lncRNA expression levels with the clinicopathological variables in primary undifferentiated NPC patients.

\begin{tabular}{|c|c|c|c|c|}
\hline & & lnc-ZNF674-1 & lnc-AL355149.1-1 & lnc-C22orf32-1 \\
\hline & & & $P$ value & \\
\hline Gender & Number & \multirow{3}{*}{0.108} & \multirow{3}{*}{$0.028^{*}$} & \multirow{3}{*}{$0.033^{*}$} \\
\hline Male & 32 & & & \\
\hline Female & 10 & & & \\
\hline \multicolumn{5}{|l|}{ Age } \\
\hline$<49$ & 19 & \multirow{2}{*}{0.733} & \multirow{2}{*}{0.970} & \multirow{2}{*}{0.810} \\
\hline$>49$ & 23 & & & \\
\hline \multicolumn{5}{|l|}{ T stage } \\
\hline $\mathrm{T} 1$ & 11 & \multirow[t]{2}{*}{$0.030^{*}$} & \multirow[t]{2}{*}{0.062} & \multirow[t]{2}{*}{$0.019^{*}$} \\
\hline $\mathrm{T} 2-4$ & 31 & & & \\
\hline \multicolumn{5}{|l|}{ Nodal status } \\
\hline Negative & 7 & \multirow[t]{2}{*}{0.895} & \multirow{2}{*}{0.692} & \multirow[t]{2}{*}{0.692} \\
\hline Positive & 35 & & & \\
\hline
\end{tabular}

${ }^{*} P$ value below 0.05 was considered statistically significant.

the same recurrent NPC patients (Figure 4). lnc-BCL2L11-3 was found to be upregulated in the recurrent NPC tissues in comparison with the paired normal tissues $(P=0.002$, Wilcoxon Signed Rank test). For lnc-AL355149.1-1 and lncZNF674-1, high expression was found in primary NPC. However, significant expression reduction was observed in the recurrent NPC tissues $(P=0.022$ and 0.002 , respectively, Wilcoxon Signed Rank test).

\section{Discussion}

The differential expression of lnc-AL355149.1-1, lncC22orf32-1, and lnc-ZNF674-1 observed in NPC might be attributed to epigenetic regulation including microRNA, DNA methylation, and histone modification. As predicted by a bioinformatics tool MirTarget2 (http://mirdb.org/miRDB/ index.html), lnc-AL355149.1-1 is targeted by hsa-miR-939, hsa-miR-4283, and hsa-miR-3191-5p; lnc-C22orf32-1 is targeted by hsa-miR-4751; lnc-ZNF674-1 is targeted by hsamiR-1296, hsa-miR-1292, and hsa-miR-3179. In addition, DNA methylation and histone modification may also contribute to the aberrant expression of these lncRNAs. For example, DNA methylation, H3K4 histone methylation, and H3K9 histone methylation are found in lncAL355149.1-1 gene in different human cell lines (lncRNome, http://genome.igib.res.in/lncRNome/).

lncRNAs could exert biological functions by interacting with RNA or protein molecules. An lncRNA, highly upregulated in liver cancer (HULC), could bind to miR-372, thus modulating the expression of miR-372 at posttranscriptional level [13]. IncRNA HOTAIR interacted with polycomb chromatin remodeling complex 2 (PRC2) by its $5^{\prime}$ domain and bind to LSD1/CoREST/REST complex via its $3^{\prime}$ domain, resulting in a specific histone methylation pattern [14]. To explore the possible model by which these differentially expressed lncRNAs exert their biological functions, we tried to predict their binding capacity via database lncRNome. The nucleotides 595-597 of lnc-ZNF674-1 are predicted to be protein binding sites, indicating that it may play a role in NPC by interacting with specific proteins.

Primary NPC is highly sensitive to radiotherapy and the use of concurrent chemoradiotherapy has significantly improved the local control rates of NPC [15]. Nevertheless, local recurrence still appears in approximately $10-30 \%$ of patients after primary radiotherapy [16]. Since recurrent NPC is possibly derived from those NPC cells that could escape from radiotherapy, the recurrent NPC might exhibit distinctive mRNA, miRNA, and protein expression patterns in comparison with primary NPC. For instance, the expression levels of miR-98, TR- $\alpha 2$ mRNA, multidrug resistance 1 (MDR-1) protein, and glutathione-S-transferase- $\pi$ (GST- $\pi$ ) protein were different between recurrent NPC and primary NPC $[17,18]$. Likewise, in this study, we also observed a diverse lncRNA expression pattern between recurrent NPC and primary NPC. For example, the aberrant expression of lnc-BCL2L11-3 was only observed in recurrent NPC but not in primary NPC, suggesting that lncRNAs might play distinct roles in the initiation and progression of recurrent NPC and primary NPC.

According to Global Cancer Statistics [19], NPC is a maledominant disease with incidence rates usually 2 to 3 times lower in females. In our cohort, the ratio between males and females is about 3 . Therefore, one of the limitations of this study is the small sample size of females. Another concern about our patient samples is the large age range. To investigate whether the large age range would affect the expression level of lncRNAs, we divided our patients into two groups according to various cutoff values of age. There are no significant differences in the expression of lncRNAs between the two groups of patients divided by varied cutoff values of age. 

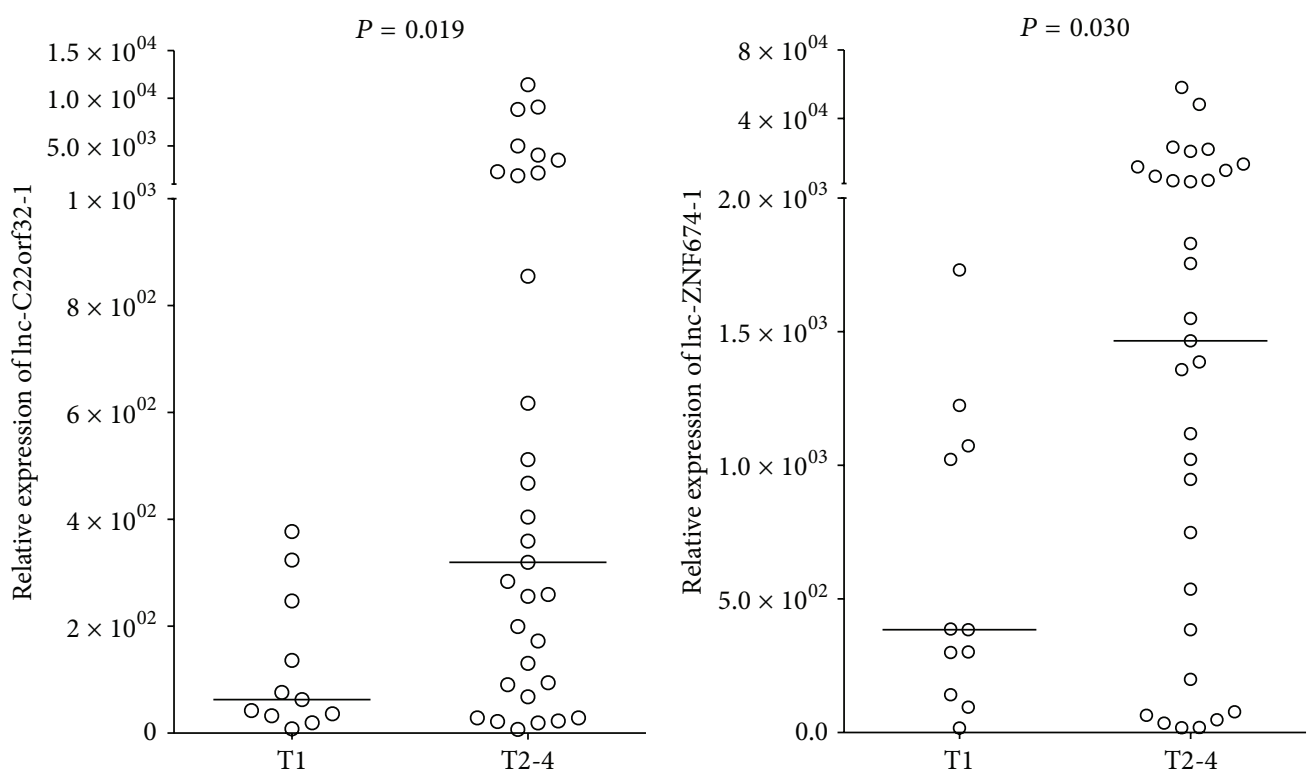

(a)
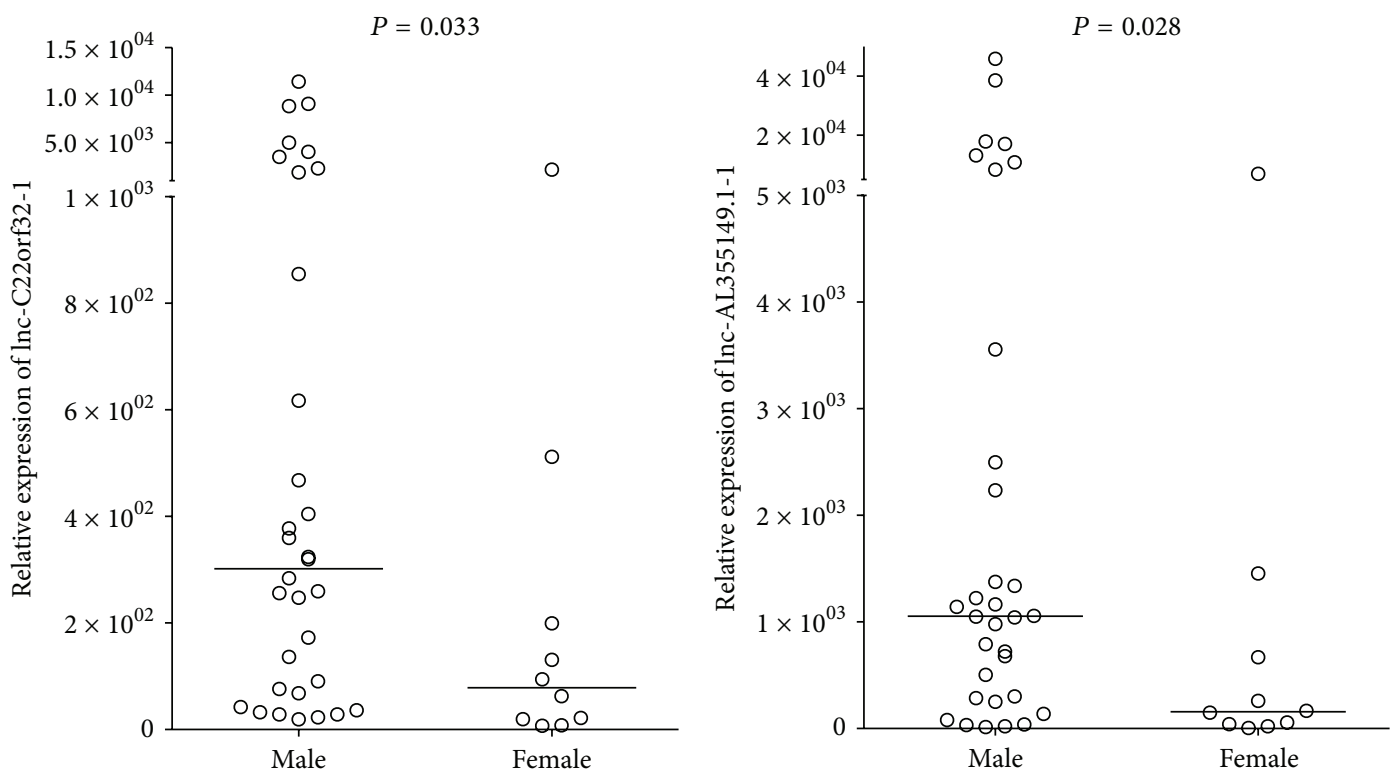

(b)

FIGURE 3: Relative expression levels of lncRNAs in primary NPC patients with different T stage (a) and gender (b). The relative expression levels were normalized to GAPDH by qPCR analysis. The difference was calculated using the Mann-Whitney $U$ test.

The differential lncRNA expression patterns could be represented by the microarray probsets, which were originally intended to map the somatic gene expression level. This approach has proven successful in identifying the altered lncRNA expression in the nucleus accumbens of heroin abusers. Michelhaugh SK reanalyzed the existing Affymetrix microarray dataset and identified the $5 \operatorname{lncRNAs}$ dysregulated in the heroin abusers [10]. They curated existing RNA databases including RNAdb and H-Invitational to generate a list of lncRNA and later matched them with the probeset on the Affymetrix U133A and U133B chips [10]. Using similar approaches, we demonstrated that the differential lncRNA expression data could possibly be retrieved from the existing microarray dataset for use, to identify candidate lncRNA which is implicated in the pathogenesis of NPC.

\section{Conclusions}

Our results demonstrated that it is feasible to identify the differentially expressed lncRNA in the microarray dataset by functional reannotation. The association of lncRNA with gender and tumor size implicated that lncRNA possibly plays a part in the pathogenesis of primary NPC. Further, 


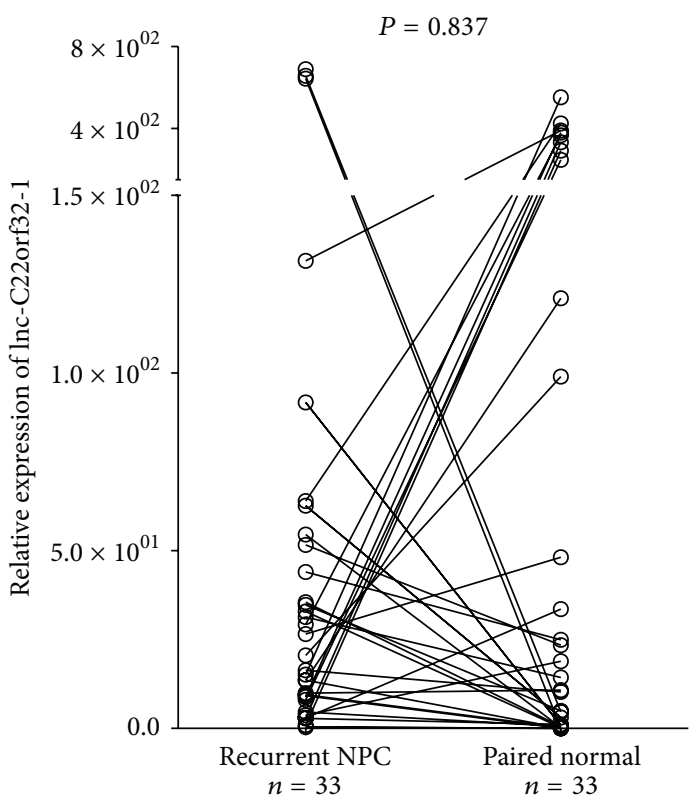

(a)

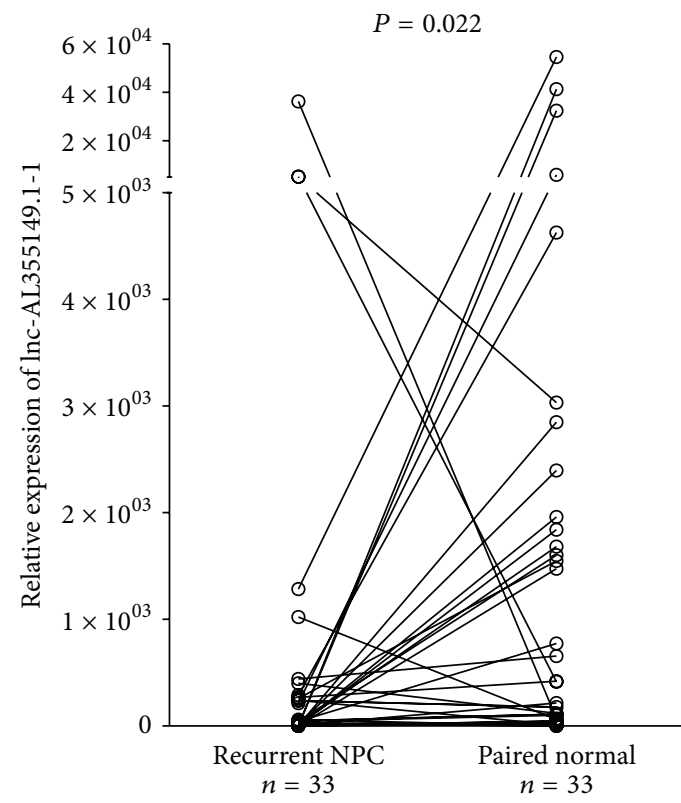

(c)

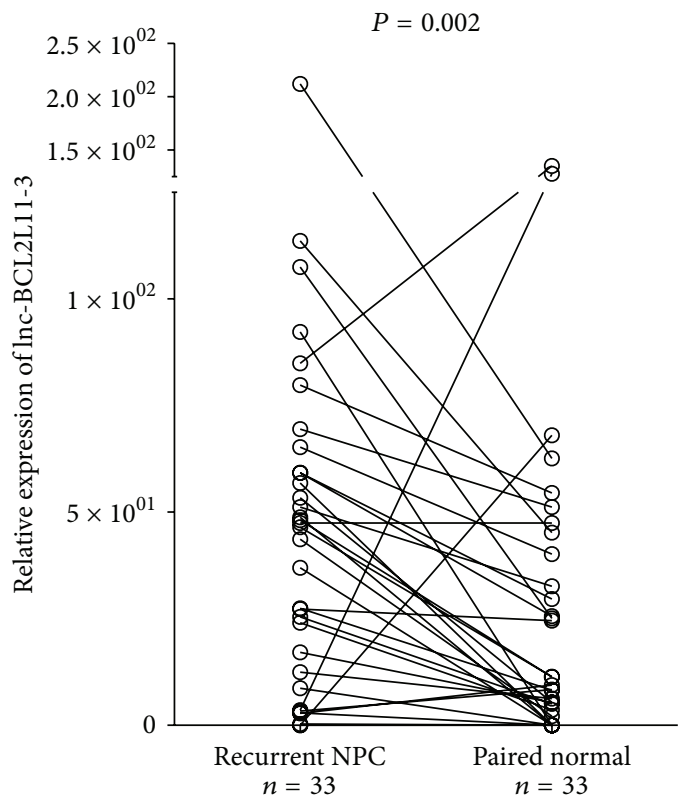

(b)

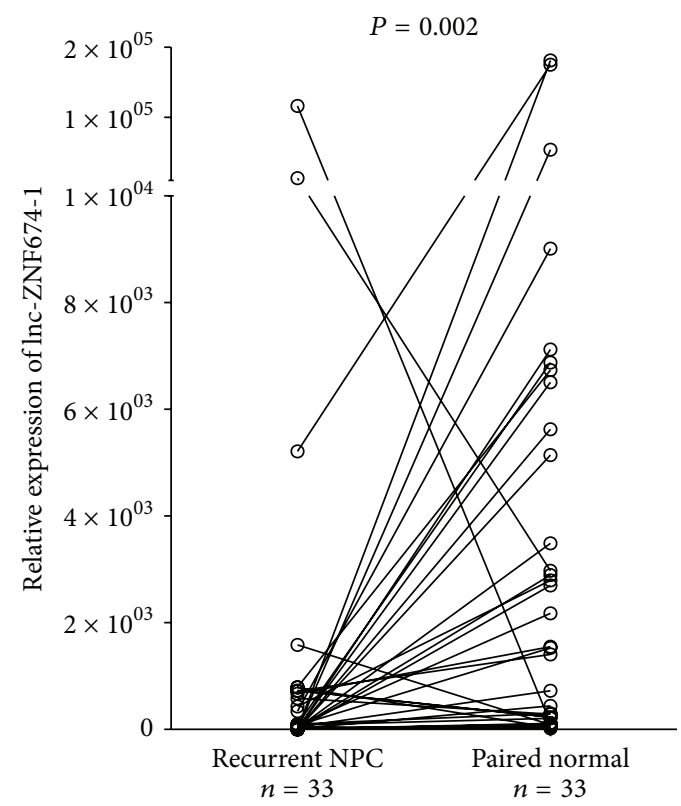

(d)

FIGURE 4: Relative expression levels of lncRNAs in paired tumor and normal tissue samples from patients with recurrent NPC. The relative expression levels were normalized to GAPDH by qPCR analysis. The difference between tumor and normal was calculated using the Wilcoxon Signed Rank test.

the distinctive lncRNA identified in the recurrent NPC may reveal a distinctive development mechanism underlying tumor recurrence. Further studies are warranted to explore the pathological significance of lncRNA in the development of NPC.

\section{Conflict of Interests}

The authors declare that there is no conflict of interests regarding the publication of this paper.

\section{Acknowledgments}

This study was supported by Seed Funding of Basic Research, The University of Hong Kong, and Hong Kong Area of Excellent Scheme, Hong Kong UGC.

\section{References}

[1] E. T. Chang and H.-O. Adami, "The enigmatic epidemiology of nasopharyngeal carcinoma," Cancer Epidemiology Biomarkers and Prevention, vol. 15, no. 10, pp. 1765-1777, 2006. 
[2] J. E. Marks, J. L. Phillips, and H. R. Menck, "The national data base report on the relationship of race and national origin to the histology of nasopharyngeal carcinoma," Cancer, vol. 83, pp. 582-588, 1998.

[3] W. I. Wei and J. S. T. Sham, "Nasopharyngeal carcinoma," The Lancet, vol. 365, no. 9476, pp. 2041-2054, 2005.

[4] W. I. Wei, J. Y. W. Chan, R. W. M. Ng, and W. K. Ho, "Surgical salvage of persistent or recurrent nasopharyngeal carcinoma with maxillary swing approach-critical appraisal after 2 decades," Head and Neck, vol. 33, no. 7, pp. 969-975, 2011.

[5] M. Huarte and J. L. Rinn, "Large non-coding RNAs: missing links in cancer?" Human Molecular Genetics, vol. 19, no. 2, pp. R152-R161, 2010.

[6] J. R. Prensner and A. M. Chinnaiyan, "The emergence of IncRNAs in cancer biology," Cancer Discovery, vol. 1, no. 5, pp. 391-407, 2011.

[7] R. J. Taft, K. C. Pang, T. R. Mercer, M. Dinger, and J. S. Mattick, "Non-coding RNAs: regulators of disease," Journal of Pathology, vol. 220, no. 2, pp. 126-139, 2010.

[8] R. A. Gupta, N. Shah, K. C. Wang et al., "Long non-coding RNA HOTAIR reprograms chromatin state to promote cancer metastasis," Nature, vol. 464, no. 7291, pp. 1071-1076, 2010.

[9] Y. Nie, X. Liu, S. Qu, E. Song, H. Zou, and C. Gong, "Long noncoding RNA HOTAIR is an independent prognostic marker for nasopharyngeal carcinoma progression and survival," Cancer Science, vol. 104, no. 4, pp. 458-464, 2013.

[10] S. K. Michelhaugh, L. Lipovich, J. Blythe, H. Jia, G. Kapatos, and M. J. Bannon, "Mining affymetrix microarray data for long noncoding RNAs: altered expression in the nucleus accumbens of heroin abusers," Journal of Neurochemistry, vol. 116, no. 3, pp. 459-466, 2011.

[11] Q. Liao, H. Xiao, D. Bu et al., "NcFANs: a web server for functional annotation of long non-coding RNAs," Nucleic Acids Research, vol. 39, no. 2, pp. W118-W124, 2011.

[12] L. E. Dodd, S. Sengupta, I.-H. Chen et al., "Genes involved in DNA repair and nitrosami ne metabolism and those located on chromosome $14 \mathrm{q} 32$ are dysregulated in nasopharyngeal carcinoma," Cancer Epidemiology Biomarkers and Prevention, vol. 15, no. 11, pp. 2216-2225, 2006.

[13] J. Wang, X. Liu, H. Wu et al., "CREB up-regulates long non-coding RNA, HULC expression through interaction with microRNA-372 in liver cancer," Nucleic Acids Research, vol. 38, no. 16, pp. 5366-5383, 2010.

[14] M.-C. Tsai, O. Manor, Y. Wan et al., "Long noncoding RNA as modular scaffold of histone modification complexes," Science, vol. 329, no. 5992, pp. 689-693, 2010.

[15] S.-P. Hao and N.-M. Tsang, "Surgical management of recurrent nasopharyngeal carcinoma," Chang Gung Medical Journal, vol. 33, no. 4, pp. 361-369, 2010.

[16] D. T. T. Chua, J. S. T. Sham, D. L. W. Kwong, W. I. Wei, G. K. H. Au, and D. Choy, "Locally recurrent nasopharyngeal carcinoma: treatment results for patients with computed tomography assessment," International Journal of Radiation Oncology Biology Physics, vol. 41, no. 2, pp. 379-386, 1998.

[17] N. M. Alajez, W. Shi, A. B. Y. Hui et al., "Enhancer of Zeste homolog $2(\mathrm{EZH} 2)$ is overexpressed in recurrent nasopharyngeal carcinoma and is regulated by miR-26a, miR-101, and miR98," Cell Death and Disease, vol. 1, no. 10, article e85, 2010.

[18] C.-L. Chen, T.-S. Sheen, I.-U. Lou, and A.-C. Huang, "Expression of multidrug resistance 1 and glutathione-S-transferase- $\pi$ protein in nasopharyngeal carcinoma," Human Pathology, vol. 32, no. 11, pp. 1240-1244, 2001.
[19] A. Jemal, F. Bray, M. M. Center, J. Ferlay, E. Ward, and D. Forman, "Global cancer statistics," CA Cancer Journal for Clinicians, vol. 61, no. 2, pp. 69-90, 2011. 


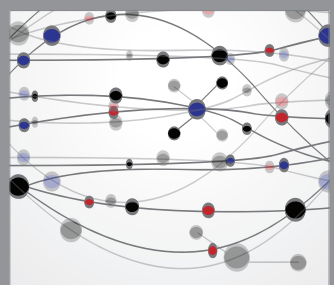

The Scientific World Journal
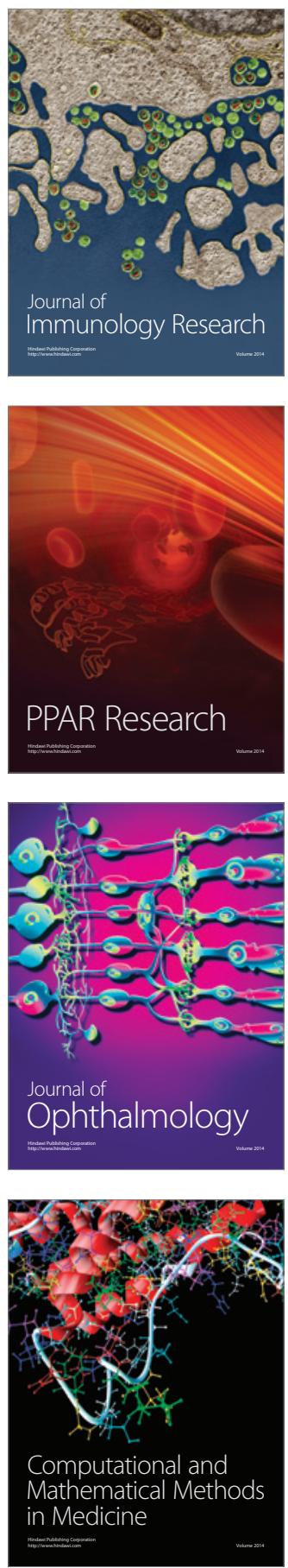

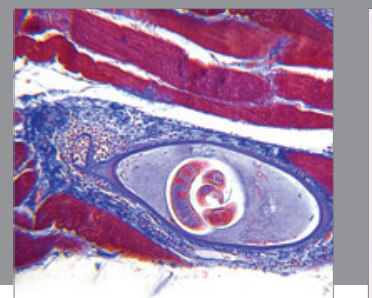

Gastroenterology

Research and Practice
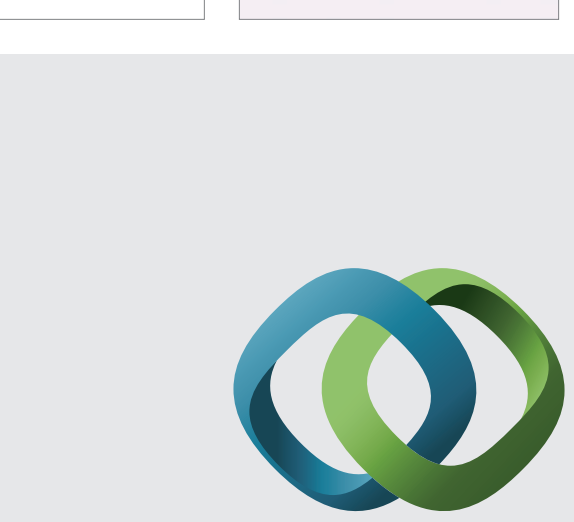

\section{Hindawi}

Submit your manuscripts at

http://www.hindawi.com
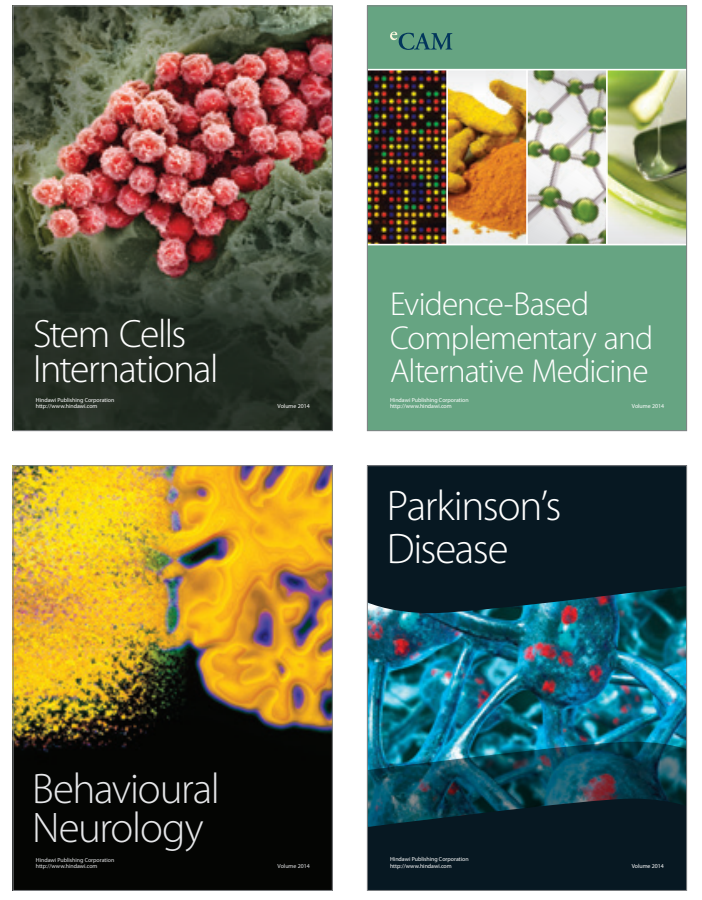
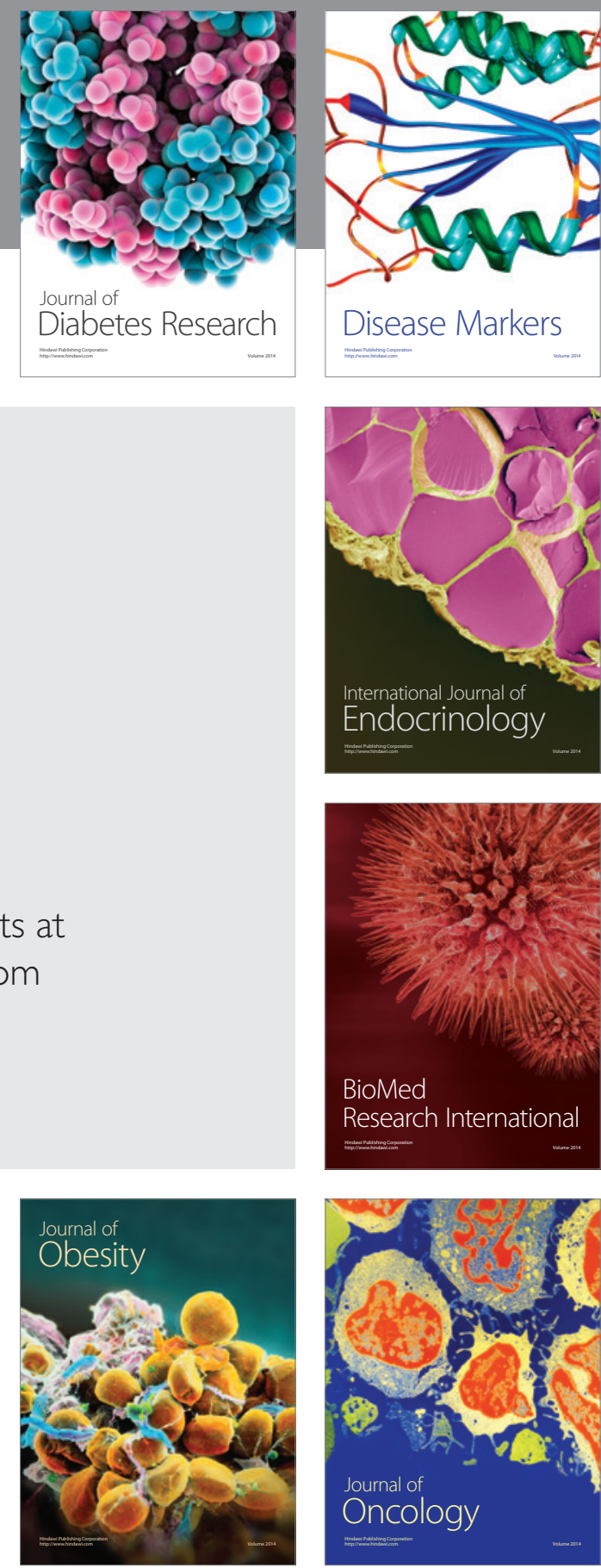

Disease Markers
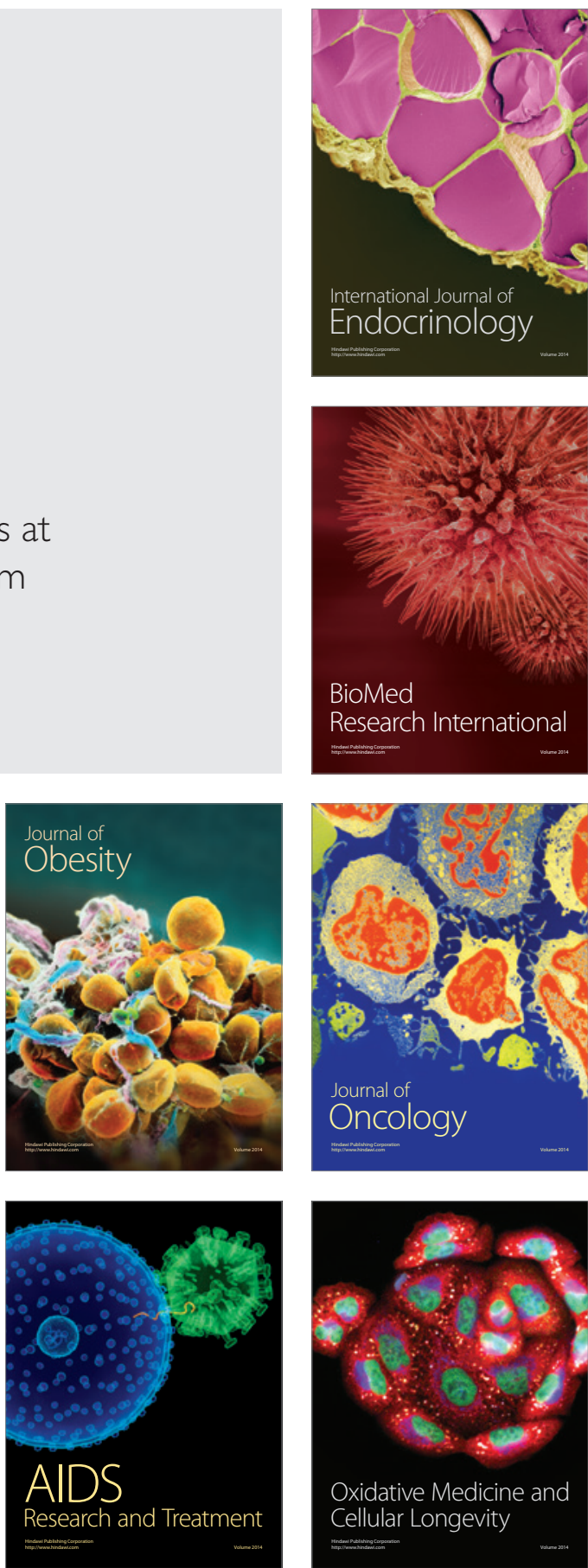